\title{
Malignant mixed Mullerian tumour of the prolapsed cervix: A case report
}

\author{
ANTHONY N. MASSINDE ${ }^{1,2^{*}}$, RICHARD R. RUMANYIKA ${ }^{1}$, ALBERT KIHUNRWA ${ }^{1}$, PETER \\ RAMBAU $^{2}$ and MOKE MAGOMA ${ }^{1}$ \\ ${ }^{1}$ Department of Obstetrics \& Gynaecology, Bugando Medical Centre, Box 1370, Mwanza, Tanzania \\ ${ }^{2}$ The Catholic University of Health and Allied Sciences, P.O. Box 1464, Mwanza, Tanzania
}

\begin{abstract}
Malignant mixed Mullerian tumour is a rare gynaecological tumour commonly presenting with vaginal bleeding, abdominal pain or mass in the uterine cavity, cervix or vagina. The neoplasms are commonly seen in postmenopausal women although it has been observed in younger women. Ovaries and the corpus of the uterus are commonly involved, whereas involvement of the cervix and vagina is rare. A 37 year-old Tanzania lady para 7 with a previous history of two genital polypectomies presented with history of recurrent vaginal mass which was associated with abnormal vaginal bleeding and foul smelling discharge. Vaginal examination revealed a prolapsed uterus with giant fungating cervical mass which was ulcerated, friable, and bled easily on touch. Impression was grade three uterine prolapse with infected cervical polyp/ cervical sarcoma. Excision of the tumour through trans-vaginal hysterectomy was performed, no lymphadenopathy was found, no adnexa abnormalities, and no involvement of the vaginal wall. Histological diagnosis of Malignant mixed Mullerian tumour of the cervix was made. Patient recovery was unremarkable; however she was lost to follow up. The patient's mass was initially suspected to be prolapsed uterus with decubitus ulcer but the histological results were of a malignant condition. Lack of clear management guidelines for some rare mixed tumours remains a challenge for clinicians in low resource settings.
\end{abstract}

Keywords: Malignant mixed Mullerian tumour, cervix, uterine prolapse, Tanzania

\section{Introduction}

Malignant mixed Mullerian tumours (MMMTs) are tumours comprised of malignant epithelial cells and malignant mesenchymal cells and they are sometimes called carcinosarcoma. These are rare gynaecological tumours commonly presenting with vaginal bleeding, abdominal pain or mass in the uterine cavity, cervix or vagina (Sharma et al., 2005; Maheshwari et al., 2006; Shi et al., 2008; Kuyumcuoglu \& Kale, 2009; Ahuja et al., 2011). The neoplasms are commonly seen in post-menopausal women although it has been observed in younger women (Una et al., 2009; Park et al., 2004). The ovaries, fallopian tubes and uterine cavity are the common sites of involvement (Kourea et al., 2008; Garcia-Galvis et al., 2008); where as cervical and vaginal involvement is rare (Maheshwari et al., 2006; Sebenik et al., 2007; Kuyumcuoglu \& Kale, 2009; Ahuja et al., 2011). There are no specific clinical features for MMMTs, and most cases are diagnosed through histological examination (Sharma et al., 2005). MMMTs can be homologous containing a carcinomatous component (from epithelia) and a sarcomatous component (from mesenchymal) tissue. All of these are normal parts of the Mullerian system. Heterologous tumours contain mesenchyma components normally not found in the uterus such as cartilage,

* Correspondence: Dr Anthony Massinde: amassinde@yahoo.com 
bone and skeletal muscles (Ali \& Wells, 1993). The tumour is naturally aggressive with a high recurrence rate and a poor prognosis (Ruiz Tovar et al., 2006; Ahuja et al., 2011). Cervical MMMT is often not clinically suspected initially. In this report, we describe a case of cervical MMMT that presented as a cervical ulcerative mass with uterine prolapse in Bugando Medical Centre in Mwanza, northern Tanzania.

\section{Case presentation}

A 37 year-old para 7 Tanzanian presented with a history of recurrent protruding mass from the vagina which was associated with abnormal vaginal bleeding and passing of odorous discharge for eight months prior to admission. She presented with a history of dizziness, headache, and awareness of her heartbeat with no history of fainting. She gave a past history of having polypectomy twice in 2009 and 2010 at peripheral hospital; but no histological results were available to confirm the histological diagnosis of the polyps. All deliveries were normal vaginal births with uneventful postpartum courses. Physical examination revealed a healthy looking woman who was not wasted and moderately pale. The abdomen was soft and nontender with no palpable mass. Vaginal examination revealed a grade three prolapsed uterus with a giant fungating, ulcerative and friable cervical mass that bled easily on touch and had malodorous discharge. The mass measured $6 \times 8 \mathrm{~cm}$ at the largest point (Figure1). Review of systems was unremarkable.

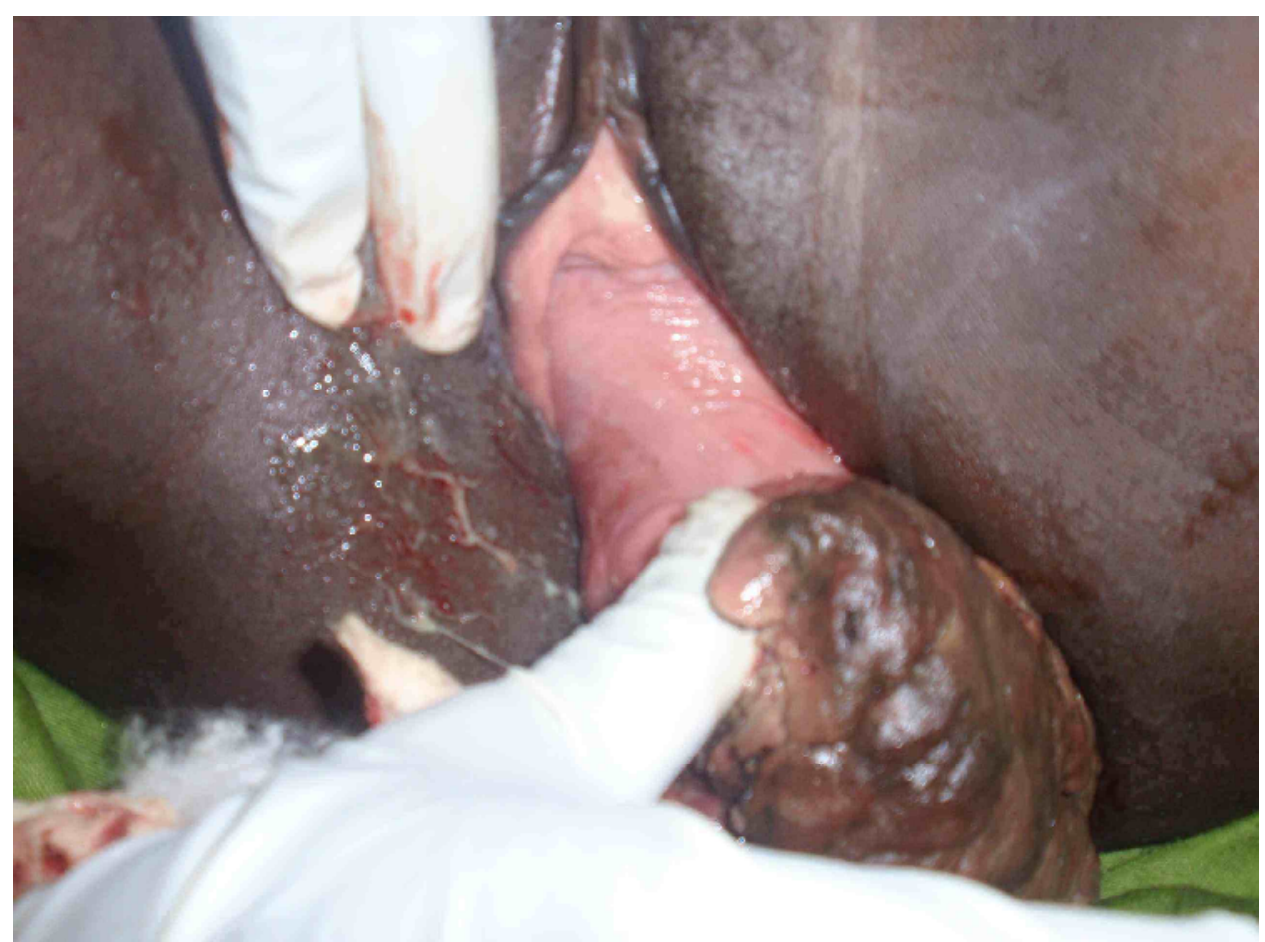

Figure 1: A giant fungating mass attached to prolapsed cervix

Investigations done included haemoglobin of $7.3 \mathrm{~g} / \mathrm{dl}$ and blood grouping and cross match with blood group A positive. Subsequently, she was transfused two units of whole blood. An abdominal ultrasound showed a retro-positioned uterus measuring 
$8 \times 3 \mathrm{~cm}$, normal adnexal organs and no para-aortic lymphnodes. Chest $\mathrm{x}$-ray was normal. Impression was prolapsed uterus and infected cervical polyp of uncertain nature/ cervical sarcoma.

The patient was counselled and agreed to hysterectomy, with sparing of the ovaries. Excision of the tumour through trans-vaginal hysterectomy was done. There was no lymphadenopathy, adnexa abnormalities, or involvement of the vaginal wall (anterior and posterior). Patient recovery was unremarkable. She was discharged four days after surgery with instructions for follow-up but she was lost to follow up.

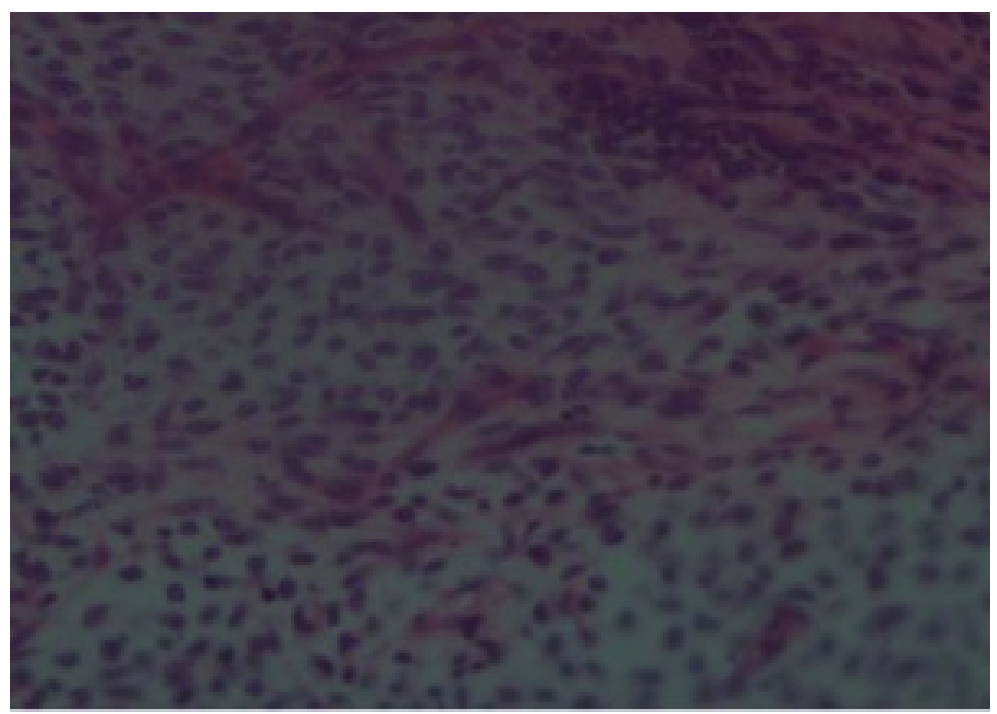

Figure 2: $\mathrm{H}$ and $\mathrm{E}$ showing sarcomatous area resembling endometrial stroma (x20)

\section{Pathological findings}

Gross examination revealed a uterus of $7 \times 5 \times 4 \mathrm{~cm}$ in largest diameter with no adnexia, a polypoid mass of $10 \times 9 \times 6 \mathrm{~cm}$ attached to the cervix. Cut section the mass was solid, fleshy whitish to greyish with areas of haemorrhage. Grossly, no extension to the endometrium or myometrium was observed and the rest of the uterus was normal. Histologically, haematoxylin and eosin stain showed a tumour comprised of low grade sarcomatous areas with round to spindle shaped cells resembling endometrial stroma, and few malignant elements with glandular features, areas with cartilage formation made up of low grade chondrocytes and areas of high grade poorly differentiated cells with rabdomyoblastic features (Figures 2, 3 and 4). The tumour was entirely confined to the cervix with no invasion to the myometrium or endometrium. With these histological findings the morphological diagnosis of malignant mixed Mullerian tumour of the cervix was made. Clinical stage one of MMMT of the cervix was the final diagnosis. 


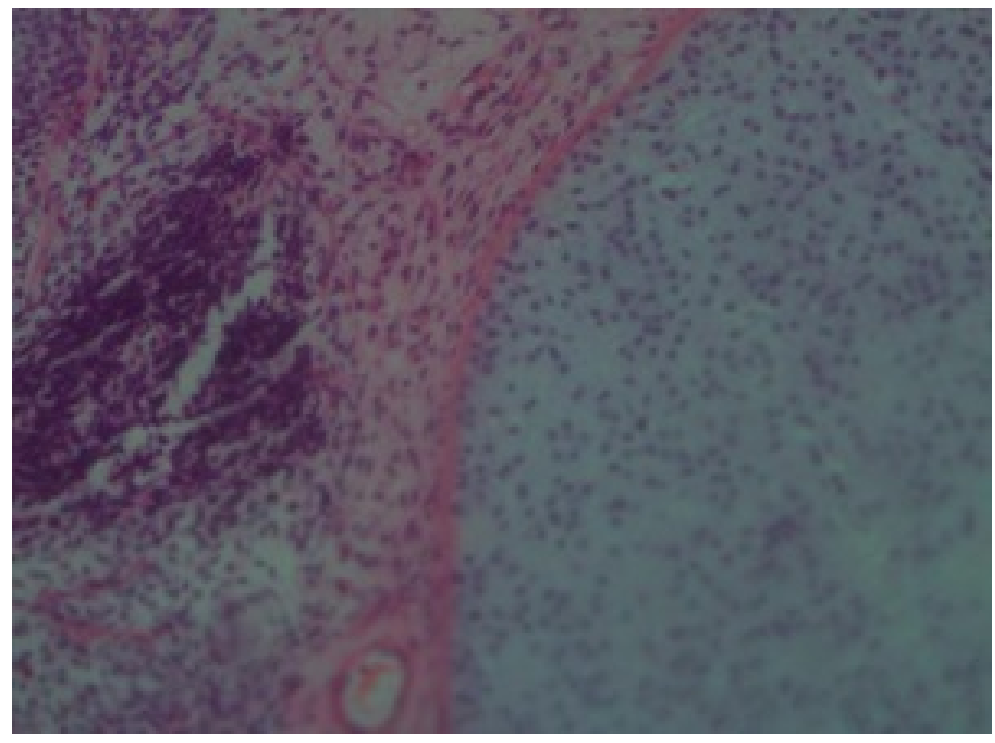

Figure 3: $\mathrm{H}$ and $\mathrm{E}$ showing sarcomatous area and cartilaginous differentiation (x10)

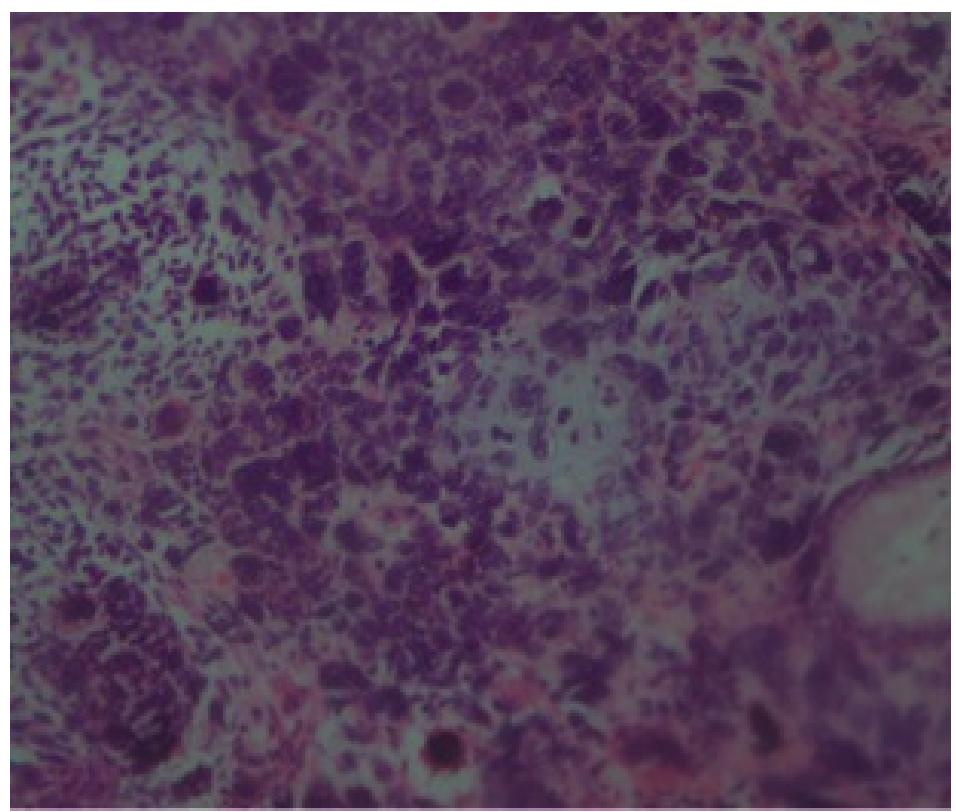

Figure 4: $\mathrm{H}$ and $\mathrm{E}$ showing area of rhabdomyoblast differentiation and component of cartilage $(x 10)$

\section{Discussion}

Malignant mixed Mullerian tumours are commonly seen in post-menopausal women and they are very aggressive with poor prognosis (Park et al., 2004; Maheshwari et al., 2006). Histological features were found to be biphasic with an epithelial component (endometrial glandular tissue) and a sarcomatous (cartilage and rhabdomyoblasts) component of extreme variability, which is important for diagnosis of MMMT (Maheshwari et al., 2006; Ahuja et al., 2011). Several other epithelial histological sub-types within sarcomatous components either alone, or in combination, have been described in the literature, such as squamous cell carcinoma, 
adenocarcinoma, adeno-squamous carcinoma, basaloid squamous carcinoma, adenoid-basal carcinoma, adenoid-cystic carcinoma and undifferentiated carcinoma (Maheshwari et al., 2006; Ahuja et al., 2011.

Management of this type of tumour is challenging, especially in poor resource settings, as this is a rare disease and its treatment is hardly described in standard gynaecological textbooks (Sharma et al., 2005; Maheshwari et al., 2006). As a result, clear management guidelines as well as survival prediction after treatment often lack. Ultrasound or Magnetic Resonance Imaging is useful in this case to exclude prolapsed mass from uterine cavity. There is reported case which appeared to be a sarcomatous mass of the uterus associated with uterine inversion (Hanprasertpong et al., 2004), although the mass seem to be attached to cervix it may actually be arising from uterine cavity. Additionally, lymphogram is useful in excluding lymphnodes involvement within the pelvis and abdominal cavity.

A few characteristics of this case need to be highlighted. The patient had two previous operations for recurrent genital polyps, but no histological specimen was taken. Recurrence should always raise the suspicion of malignant tumour and histological specimen would have been helpful in making the right diagnosis.

After correcting the anaemia, our management approach to this patient was trans-vaginal hysterectomy, skipping biopsy of the lesion for three reasons. First, initial assessment showed that the tumour was confined to the cervix, bled easily on touch, and friable. An attempt to biopsy the tumour would possibly provoke uncontrollable bleeding (Dane et al., 2009; Massinde et al., 2012), Secondly, due to limited resources, we were not able to do frozen section at the time of the surgery, and normal biopsy results would take at least two weeks to be completed. Thus, waiting for two more weeks to confirm diagnosis would have been difficult as the mass was prolapsed and bled at every contact. Thirdly, the patient had a prolapsed uterus and completed her desired family, thus the approach provided an easy way to remove the mass and the uterus without manipulating the mass itself.

Presentation of two conditions in one patient presents a problem, as the standard way of managing cancerous lesion of the cervix becomes difficult. Similar cases of epidemoid cancer associated with uterine prolapse have been reported where radical vaginal hysterectomy followed by pelvic radiation has been used. This differs from typical management of cancer of the cervix (da Silva et al., 2002). Pelvic radiation can be hazardous in this case. There is increased risk of visceral injury (Dane et al., 2009; Loizzi et al., 2010). Radical vaginal hysterectomy would have been ideal for this case (Loizzi et al., 2010; da Silva et al., 2002; Dane et al., 2009).

Although the patient was lost to follow up, a close follow-up of patients with MMMT is recommended as high rate of recurrence has been reported (Callister et al., 2004; Maheshwari et al., 2006; Sharma et al., 2005). The role of adjuvant radiation and chemotherapy is still unclear (Callister et al., 2004; Maheshwari et al., 2006). Other institutes have used radiotherapy for treatment of recurrence (Maheshwari et al., 2006). In case series by Sharma et al. (2005), treatment for MMMT was the same as that of epithelial malignancy and outcome was good except for one patient

In conclusion, diagnosis and subsequent choice of management options of any cervical mass like the one reported requires histological confirmation. 
Nevertheless, lack of clear management guidelines for some rare mixed tumours remain a challenge for clinicians in low resource settings

\section{Competing interests}

The authors declare that they have no competing interests.

\section{Authors' contributions}

AM received the patient and did initial work-up, did literature review, wrote the initial manuscript and reviewed all other subsequent versions of the manuscript. AK and $\mathrm{MM}$ reviewed the literature and reviewed all versions of the manuscript. RR performed the surgery and contributed to review of literature. PR performed the histological examination of the tumour, and contributed in writing the manuscript. All authors read and approved the final version of the manuscript.

\section{References}

Ahuja, A., Safaya, R., Prakash, G., Kumar, L. \& Shukla, N.K. (2011) Primary mixed mullerian tumour of the vagina--a case report with review of the literature Pathology, Research and Practice 207, 253-255.

Ali, S. \& Wells, M. (1993) Mixed Mullerian tumors of the uterine corpus: a review International Journal of Gynecological Cancer 3, 1-11.

Callister, M., Ramondetta, L.M., Jhingran, A., Burke, T.W. \& Eifel, P.J. (2004) Malignant mixed Mullerian tumors of the uterus: analysis of patterns of failure, prognostic factors, and treatment outcome International Journal of Radiation Oncology, Biology, Physics 58, 786-796.

da Silva, B.B., da Costa Araujo, R., Filho, C.P. \& Melo, J.A. (2002) Carcinoma of the cervix in association with uterine prolapse Gynecologic oncology ,84, 349-50.

Dane, B., Dane, C., Erginbas, M., Baran, S. \& Cetin, A. (2009) Verrucous carcinoma of the cervix in a case with uterine prolapse Annals of Diagnostic Pathology13, 344-346.

Garcia-Galvis, O.F., Cabrera-Ozoria, C., Fernandez, J.A., Stolnicu, S. \& Nogales, F.F. (2008) Malignant Mullerian mixed tumour of the ovary associated with yolk sac tumour, neuroepithelial and trophoblastic differentiation (teratoid carcinosarcoma) International Journal of Gynecologic Pathology 27, 515-520.

Hanprasertpong, J., Wootipoom, V. \& Hanprasertpong, T. (2004) Non-puerperal uterine inversion and uterine sarcoma (malignant mixed mullerian tumour): report of an unusual case Journal of Obstetrics and Gynaecology Research 30, 105-108.

Kourea, H.P., Adonakis, G., Androutsopoulos, G., Zyli, P., Kourounis, G. \& Decavalas, G. (2008) Fallopian tube malignant mixed mullerian tumour (carcinosarcoma): a case report with immunohistochemical profiling European Journal of Gynaecological Oncology 29, 538-542. 
Kuyumcuoglu, U. \& Kale, A. (2009) Homologous type of malignant mixed Mullerian tumour of the uterus presenting as a cervical mass Journal of China Medical Association 72, 533-535.

Loizzi, V., Cormio, G., Selvaggi, L., Carriero, C. \& Putignano, G. (2010) Locally advanced cervical cancer associated with complete uterine prolapse. European Journal of Cancer Care 19, 548-550.

Maheshwari, A., Gupta, S., Shet, T., Wuntkal, R. \& Tongaonkar, H.B. (2006) Diagnostic dilemma in a case of malignant mixed Mullerian tumour of the cervix. World Journal of Surgical Oncology 4, 36.

Massinde, A.N., Mpogoro, F., Rumanyika, R.N. \& Magoma, M. (2012) Uterine prolapse complicated with a giant cervical polyp. Journal of Lower Genital Tract Disease 16, 64-65.

Park, H.M., Park, M.H., Kim, Y.J., Chun, S.H., Ahn, J.J., Kim, C.I., Sung, S.H., Han, W.S. \& Kim, S.C. (2004) Mullerian adenosarcoma with sarcomatous overgrowth of the cervix presenting as cervical polyp: a case report and review of the literature. International Journal of Gynecological Cancer 14, 10241029.

Ruiz Tovar, J., Reguero Callejas, M.E., Arano Bermejo, J.I., Capote Armas, L.F., Gonzalez-Palacios Martinez, F. \& Cabanas Navarro, L. (2006) Malignant mixed Mullerian tumors. Clinical and Translational Oncology 8, 129-132.

Sebenik, M., Yan, Z., Khalbuss, W.E. \& Mittal, K. (2007) Malignant mixed mullerian tumour of the vagina: case report with review of the literature, immunohistochemical study, and evaluation for human papilloma virus Human Pathology 38, 1282-1288.

Sharma, N.K., Sorosky, J.I., Bender, D., Fletcher, M.S. \& Sood, A.K. (2005) Malignant mixed mullerian tumour (MMMT) of the cervix. Gynecologic Oncology 97, 442-445.

Shi, Y., Liu, Z., Peng, Z., Liu, H., Yang, K. \& Yao, X. (2008) The diagnosis and treatment of Mullerian adenosarcoma of the uterus. Australian and New Zealand Journal of Obstetric and Gynaecology 48, 596-600.

Una, E., Garcia-Tejeiro, M. \& Alvarez, M. (2009) Primary peritoneal malignant mixed Mullerian tumor in a young woman: achieving the best clinical benefit Case Reports in Oncology 2, 162-167. 